\section{Trajetória da política de atenção básica à saúde no Distrito Federal, Brasil (1960 a 2007): análise a partir do marco teórico do neo-institucionalismo histórico}

\author{
A history of primary health care policy in the \\ Federal District, Brazil (1960-2007): an analysis \\ based on the theoretical framework of \\ historical neo-institutionalism
}

\author{
${ }_{1}$ Universidade Católica de \\ Brasília, Brasília, Brasil. \\ 2 Secretaria de Estado da \\ Saúde do Distrito Federal, \\ Brasília, Brasil. \\ 3 Universidade de Brasília, \\ Brasília, Brasil. \\ 4 Escola de Enfermagem, \\ Universidade Federal de \\ Minas Gerais, Belo Horizonte, \\ Brasil. \\ Correspondência \\ L. B. D. Göttems \\ Universidade Católica \\ de Brasília. \\ SHCES Q. 1109, Bloco D, \\ apto. 101, Brasília, DF \\ 70658-190, Brasil. \\ leilad@ucb.br \\ leila.gottems@uol.com.br
}

\begin{abstract}
This article analyzes the history of primary health care policy in the Federal District, Brazil, based on the theoretical framework of historical neo-institutionalism, identifying the predominant configurations and trends in the various administrations of the State Health Secretariat (SES-DF) from 1960 to 2007. The study indicates that the characteristics of the Federal District's health policy are dependent on the history of the original health system plans for setting priorities and goals, as well as for the health system's implementation. This influence, in addition to the centralization of decision-making processes and limited political participation, can contribute to making primary care ancillary to hospital care, thus jeopardizing its potential to produce change in the health care model.
\end{abstract}

Health Policy; Primary Health Care; Single Health System
Leila Bernardo Donato Göttems ${ }^{1,2}$ Maria do Socorro Nantua Evangelista 1,3 Maria Raquel Gomes Maia Pires 4 Aline Ferreira Melgaço da Silva 1,2 Priscila Avelino da Silva 1,2

\section{Introdução}

O Sistema Único de Saúde (SUS), após ser instituído pela Constituição Federal brasileira de 1988 e pela Lei $n^{o} .8080 / 901$, iniciou um lento processo de reorganização da atenção à saúde na tentativa de vencer os desafios decorrentes da transição epidemiológica, das desigualdades sociais e das dificuldades encontradas pelo Estado para garantir serviços de qualidade à população. Destacam-se como obstáculos à concretização da política pública apregoada a instabilidade do financiamento, a hegemonia do modelo hospitalocêntrico em detrimento da atenção primária à saúde, a dicotomia na oferta de serviços, bem como os conflitos entre os grupos de interesse público e privado. Nesse particular, ressalta-se que o predomínio do setor privado na prestação de serviços de média e alta complexidades, de maior custo, enquanto o setor público se responsabiliza pela oferta de Atenção Básica à Saúde, menos onerosa, constitui-se num ponto estratégico para a reorientação da circulação de capital em favor do bem público ${ }^{2}$.

Emergiu da agenda política dos últimos 15 anos a proposta de organização da Atenção Primária à Saúde ou Atenção Básica à Saúde - termos aqui empregados como sinônimos - por meio da Estratégia Saúde da Família. Trata-se de um conjunto de ações, no âmbito da saúde individual e coletiva, que abrange a promoção e a proteção, a prevenção de agravos, o diagnóstico, 
o tratamento, a reabilitação e a manutenção da saúde ${ }^{3}$. Essa estratégia de organização tem caráter participativo, pressupõe a integração das ações e fundamenta-se nos princípios do SUS, enfatizando-se o trabalho em equipe, a territorialização, a co-responsabilidade sanitária e o foco nas necessidades da população, tendo as unidades básicas de saúde como contato preferencial dos usuários ao sistema de saúde 2 .

Avaliações sobre a implantação da Estratégia Saúde da Família no Brasil 4,5,6,7 revelam resultados positivos, sobretudo nos municípios de pequeno porte, com menos de 100 mil habitantes. Verifica-se elevação nas taxas de cobertura assistencial no curto prazo e melhoria no acesso da população aos serviços de saúde. Todavia, no interior do país, constata-se a persistência de antigos problemas, tais como: insuficiente oferta de profissionais de saúde, em especial médicos, enfermeiros e odontólogos, alto custo na manutenção das equipes e precário suporte ao trabalho de forma a assegurar o acesso aos demais níveis de atenção (média e alta complexidades). Por sua vez, a consolidação da Estratégia Saúde da Família nos grandes centros urbanos, sobretudo naqueles com mais de 500 mil habitantes, é complexa. Observam-se aspectos multifatoriais, como elevada complexidade epidemiológica, adensamento populacional, processo de urbanização desorganizado, desigualdades sociais e déficit em saneamento que dificultam a implantação da Estratégia Saúde da Família nesses locais 7 .

Nesse contexto, o sistema de saúde do Distrito Federal, com características semelhantes às das grandes metrópoles, constitui um importante exemplo para a análise das repercussões de tais iniciativas sobre a reorganização da Atenção Primária à Saúde. Situado na Região Integrada de Desenvolvimento do Distrito Federal e Entorno (RIDE), delimitado pelos municípios dos estados de Goiás e de Minas Gerais e inaugurado em 1960, o Distrito Federal possuía, em 2005, uma população estimada pelo Instituto Brasileiro de Geografia e Estatística (IBGE) de 2,4 milhões de habitantes ${ }^{8}$. Integra o sistema de saúde uma ampla rede própria, com oferta de atenção em todos os níveis de complexidade (Atenção Primária à Saúde, média e alta complexidades). Os leitos hospitalares públicos da Secretaria de Estado da Saúde do Distrito Federal (SES-DF) equivalem a $83 \%$ do total de leitos SUS, que representam $70,4 \%$ do total disponível no Distrito Federal (Cadastro Nacional de Estabelecimentos de Saúde. http://cnes.datasaus.gov.br). Em relação à Atenção Primária à Saúde, há extensa rede física regionalizada, em média uma unidade básica de saúde para cada 25 mil habitantes ${ }^{3}$. Todavia, a co-existência na atenção básica do modelo tradicional, da Estratégia Saúde da Família e do Programa de Agentes Comunitários de Saúde (PACS) limitam a reorganização proposta ${ }^{3}$. Atualmente, a cobertura populacional pela Estratégia Saúde da Família é de $8,7 \%$ e pelo PACS de $15,4 \%$, totalizando $24,1 \%$, conforme dados fornecidos pela SES-DF.

O tema central do presente estudo é a identificação da forma como a Atenção Primária à Saúde vem sendo abordada na política de saúde do Distrito Federal e dos problemas que persistem ao longo da história. Parte-se da hipótese de que a trajetória dessa política foi marcada por maciços investimentos no modelo hospitalocêntrico, com a presença de instituições formais e informais que se interpõem às mudanças que poderiam fortalecer a capacidade resolutiva da Atenção Primária à Saúde. Tem-se por objetivos: (1) analisar a trajetória das políticas de saúde a partir da identificação "espaço-temporal" da Atenção Primária à Saúde no Distrito Federal; (2) identificar as tendências predominantes nas diferentes gestões do sistema de saúde local, considerando os marcos teóricos do neo-institucionalismo e da dependência de trajetória ("path dependence").

\section{Metodologia}

Trata-se de pesquisa teórica e documental, integrante de projeto de tese de doutorado, com enfoque qualitativo, orientada para a reconstrução de teorias, quadros de referência, condições explicativas da realidade, polêmicas e discussões pertinentes 9 . A revisão da literatura parte de textos científicos publicados em periódicos e disponíveis nas bibliotecas virtuais, especialmente a Biblioteca Virtual em Saúde (http: / / www.bireme. br) e Scientific Electronic Library Online (http:// www.scielo.br), com o uso das palavras-chave políticas públicas, neo-institucionalismo, "path dependence”, política de saúde, e expressõeschave atenção básica e/ou atenção primária à saúde. Para sistematização dos dados utilizou-se a análise documental 10 dos relatórios de gestão, dos planos de saúde e das publicações sobre a política de saúde local, disponíveis em acervo físico na rede de bibliotecas da SES-DF. As informações factuais estruturam-se nas seguintes categorias: organização dos serviços de média e alta complexidades; organização da Atenção Primária à Saúde; fortalecimento de recursos humanos em saúde; gestão do sistema de saúde, sintetizada por meio da análise de conteúdo, que consiste nas "constantes idas e vindas da teoria ao material de análise, do material de análise à teoria, e pressupõe a elaboração de várias versões do 
sistema categórico" 11 (p. 58). A pesquisa é parte integrante da investigação Avaliação da Atenção Básica no DF: Estudo sobre Organização, Acesso e Resolubilidade dos Serviços de Saúde, financiada parcialmente com recursos do Conselho Nacional de Desenvolvimento Científico e Tecnológico (CNPq, processo no. 402842/2005-7) e aprovada pelo Comitê de Ética em Pesquisa da SES-DF (parecer $n^{\circ} .219 / 2008$ ).

\section{O neo-institucionalismo na análise de políticas públicas}

A análise de políticas públicas é, por definição, a avaliação do governo em ação ou o estudo do conjunto de ações que um governo realiza para alcançar objetivos em relação a um determinado problema ou conflito social 12,13. O enfoque do neo-institucionalismo foi adotado para subsidiar a análise, uma vez que o modelo dispõe de ferramenta capaz de permitir uma reflexão a partir da dimensão temporal e histórica desta formulação 14,15. $\mathrm{O}$ argumento central das políticas públicas nessa abordagem refere-se à construção de parâmetros essenciais, com base em procedimentos, protocolos, normas e convenções oficiais, oficiosas e inerentes à estrutura organizacional da comunidade política.

O neo-institucionalismo destaca o papel das instituições como elemento mediador entre estruturas sociais e comportamentos individuais 16 . Permite discutir os pressupostos político-institucionais dos processos de decisão política, enfatizar a importância do fator institucional para explicação dos acontecimentos políticos concretos, além de revelar a existência de regras gerais e entendimentos prevalentes em cada sociedade, que influenciam a interpretação e o agir das pessoas 17 . As instituições, que agiriam como matrizes de reprodução social, orientando padrões de comportamento e limitando o âmbito das ações possíveis nos diferentes contextos sociais, são as regras formais e informais que não apenas estruturam a sociedade, mas também orientam as relações sociais. As regras formais emanam de leis e constituições impostas por um governo ou agente com poder de coerção, enquanto as regras informais são normas ou códigos de conduta que emergem da própria sociedade ou são impostas por determinada estrutura de poder e dominação ideológica 18. Nessa perspectiva, a principal função das instituições na sociedade é reduzir a incerteza, estabelecendo uma estrutura estável resultante da interação humana. Todavia, as instituições estão em mudança permanente, evoluem, modificam-se de maneira incremen- tal e, por conseguinte, alteram escolhas e o fazem de modo contínuo. A mudança incremental provém das percepções dos empreendedores políticos e econômicos, dependendo, portanto, da forma como as informações são recebidas e processadas 18 .

Outro conceito central no neo-institucionalismo histórico e importante para a análise é o de dependência da trajetória ("path dependen$c e$ "), que diz respeito às dimensões temporais dos processos sociais reais que permitem identificar e explicar mecanismos sociais, entendidos como "caminhos plausíveis e freqüentes de como as coisas acontecem" 18 (p. 6). A análise, nessa perspectiva, tem as seguintes características: destaca a questão do tempo e a seqüência das ações desenvolvidas; admite que conseqüências importantes possam resultar de eventos relativamente medíocres, insignificantes ou contingentes que influenciam o curso da implementação da política pública; contradiz a argumentação e a explicação das ciências sociais, que associam resultados significativos a inúmeras causas 20 . A dependência da trajetória também é definida como uma seqüência de escolhas condicionadas por opções anteriores, com tendência a reforçá-las sem que esta conseqüência seja considerada pelos agentes que tomam a decisão. Sendo assim, as opções assumidas nesses momentos tendem à permanência e, a menos que surja alguma força suficiente para superar esta inércia de origem, limitam opções posteriores 21 .

\section{A trajetória histórica da política de atenção básica no Distrito Federal (1960 a 2008)}

No presente estudo, procura-se apreender as características institucionais da política de saúde a partir das diretrizes dos planos e demais documentos oficiais, uma vez que estas revelam, explícita ou implicitamente, a orientação técnica do momento considerado. A trajetória refere-se à seqüência temporal dos processos políticos $\mathrm{e}$ aos seus impactos nos resultados atuais. Parte-se da premissa de que as características institucionais e a trajetória da política constituem categorias que refletem o conjunto de regras e acordos que nortearam a política de saúde nos diferentes momentos e podem contribuir para explicar sua conformação atual.

\section{Origem da política de saúde no Distrito Federal}

Na década de 1960, quando Brasília foi criada, o setor saúde no país se caracterizava pela bus- 
ca de novas tecnologias, associada à progressiva especialização da prática médica. Era visível o contraste entre o enfoque curativo e o preventivo, entre a assistência individual e a coletiva. Para a nova capital, além de uma estrutura arquitetônica arrojada, propunha-se a oferta de serviços públicos modernos em relação não só às demais regiões do país, mas também do mundo. Com esse propósito, buscou-se uma organização "sistêmica e integral com vistas a oferecer, universalmente, serviços eqüitativos de saúde, dentro de um modelo assistencial voltado aos diversos aspectos relacionados com a manutenção da saúde e sua pronta recuperação, quando necessário" 22 (p. 35).

Na prática, o sistema de saúde do Distrito Federal, até o presente, gravita em torno de dois principais Planos de Saúde: Bandeira de Mello de 1960 e Jofran Frejat de 1979. O primeiro propôs a criação de uma instância de gestão denominada Fundação Hospitalar do Distrito Federal (FHDF), subordinada à SES-DF e integrada por estruturas hospitalares de diferentes níveis de complexidade e uma rede de serviços básicos em todo o território, capaz de oferecer assistência a uma população de 500 mil habitantes 23 .

O Plano de Saúde Bandeira de Mello propunha uma estrutura com a seguinte configuração: (a) eliminação da multiplicidade de órgãos assistenciais; (b) distribuição de centros de saúde e hospitais por grupos populacionais; (c) redução do custo e aumento da eficiência dos serviços, resultante da concentração; (d) comodidade para a população, evitando-se deslocamentos; (e) livre arbítrio do usuário na escolha do médico que trataria da sua saúde; (f) pagamento aos médicos por produtividade; (g) regime de trabalho integral; (h) possibilidade atendimento médico a pacientes particulares; (i) participação da população na solução de problemas por meio dos Conselhos Comunitários de Saúde; (j) serviço de atendimento domiciliar a convalescentes, evitando-se a permanência em leito hospitalar 22. Além da criação de um órgão de administração central, o plano estruturava os serviços com nítida separação entre as áreas de ação dos órgãos executivos e normativos. Propunha a centralização da orientação técnica e a descentralização dos serviços de saúde em três zonas: central, intermediária e rural. O planejamento incluía a construção de um hospital de alta complexidade, 11 hospitais gerais e seis hospitais rurais, circundados por Unidades Satélites. As diretrizes visavam a atender às diversas especialidades clinicas, estimulando a rotatividade de pacientes e reduzindo a permanência hospitalar 23.

Ao final da década de 1970, cerca de $70 \%$ dos atendimentos ocorriam no pronto-socorro. A re- de física contava com seis hospitais, cinco postos de saúde e uma Unidade Integrada de Saúde, sob a responsabilidade da Universidade de Brasília 23 . A estrutura existente se tornaria insuficiente e inadequada para atender à população. Além do acelerado crescimento demográfico, a insatisfação dos usuários com o atendimento, a demanda reprimida e a limitação na qualificação dos profissionais contrastavam com a proposta do Plano de Saúde original 22.

A tentativa de solução veio com o Plano proposto por Jofran Frejat, inspirado no ideário de Alma Ata. O novo modelo apoiava-se na estratégia do cuidado primário prestado em postos rurais e nos centros de saúde, com a oferta de especialidades básicas em ginecologia, pediatria, clínica médica e odontologia para cada 30 mil habitantes. Previa, também, a construção de hospitais regionais em cada cidade satélite e um hospital de base, referência para a alta complexidade. Estabelecia a hierarquia na assistência, a referência e a contra-referência, além da regionalização dos serviços. Adicionalmente, adotou a contratação de novos profissionais de saúde, o que ampliou o acesso de usuários e a oferta de serviços em atenção básica, com conseqüente melhoria dos indicadores, particularmente, os de mortalidade infantil e materna 24 . A avaliação da proposta Frejat indicou que, em termos de infra-estrutura física, houve avanços no cuidado primário. Todavia, constatou-se limitação na formação de profissionais adequados ao modelo, resultando no "gradativo retorno da demanda espontânea aos serviços de emergência hospitalar e abandono dos centros de saúde pela população e pelos profissionais" 22 (p. 37). Contribuíram para esse regresso a dificuldade de envolvimento da comunidade na vocalização de demandas, a centralização da gestão e a baixa permeabilidade das questões sociais.

\section{- Primeiro período: 1960 a 1969}

Nesse ínterim, o Distrito Federal era governado por prefeitos nomeados diretamente pelo Governo Federal, com uma administração mais característica de município do que de estado. Dirigiram a SES-DF no período sete Secretários de Saúde, todos médicos, com uma média de 1,3 ano de permanência no cargo.

A residência médica destaca-se como importante iniciativa da organização do sistema de saúde, com ênfase nas especialidades clínicas e hospitalares, cerne da estrutura dos futuros investimentos do setor. O Plano Bandeira de Melo deu destaque à construção de unidades hospitalares, inclusive rurais, implantando uma concepção coerente com o ideário político da época, 
que privilegiava a incorporação de tecnologias na medicina, sob os influxos do paradigma hospitalocêntrico e biologicista. Em síntese, pode-se dizer que, nesse período, foram lançadas as bases da política de saúde do Distrito Federal ancorada na atenção curativa, individual e especializada, com estímulo à formação de profissionais de enfermagem de nível médio, para apoiar o trabalho médico. Concebe-se assim o embrião das dificuldades futuras para a implantação da política de atenção básica, dificuldades estas que o conceito de dependência da trajetória ajuda a desvelar (Tabela 1).

\section{- Segundo período: 1969 a 1983}

No período compreendido entre 1969 e 1983, a política de saúde do Distrito Federal foi conduzida por três Secretários de Saúde, todos com formação em medicina, com uma média de 4,6 anos de gestão cada um, o que significa baixa rotatividade na administração da saúde.

A análise das ações realizadas no período revela a primeira proposta de mudança na organização do sistema de saúde do Distrito Federal, que tinha como objeto central a atenção primária. Para consolidar o Plano Frejat foram construídas 41 unidades de atendimento, entre postos e centros de saúde. Criaram-se estruturas de apoio, propiciando a descentralização dos hospitais e a ampliação do acesso da população aos serviços. Contudo, os investimentos na infra-estrutura não foram acompanhados de mudanças na organização do processo de trabalho e na formação dos profissionais, sobretudo de médicos. Destarte, o plano não provocou mudanças na organização do modelo assistencial, reproduzindo a política anterior, na medida em que fragilizou a capacidade resolutiva da atenção básica junto à população. Essa circunstância corrobora o argumento segundo o qual as opções assumidas nos momentos de proposição de mudanças tendem à permanência e, a menos que surja alguma força suficiente para superar esta inércia de origem, limitam opções posteriores 21 . O cenário descrito mostra ações voltadas para a atenção primária, centradas em problemas prevalentes de saúde, em métodos de prevenção e controle de agravos, por meio da vigilância epidemiológica. Observam-se, ainda, iniciativas de planejamento e de avaliação das ações em nível local e até mesmo a tentativa pontual de saúde da família (Tabela 2).

\section{- Terceiro período: 1983 a 1990}

Três médicos conduziram a SES-DF no período, com uma média de permanência no cargo de 2,3 anos. Vale lembrar que somente a partir da Constituição Federal de 1988 a população do Distrito Federal passa a escolher seus representantes por meio do sufrágio universal. Tal fato tem relevância especial no caso da saúde, haja vista o caráter democrático e participativo do SUS, originado a partir de movimento social organizado, a Reforma Sanitária.

Destaca-se o espaço para vocalização das demandas da comunidade e o estímulo ao trabalho na Atenção Primária à Saúde, por meio da gratificação aos profissionais lotados nas unidades básicas de saúde. Nesse período ocorre a primeira iniciativa de debate com a população, aberta pela I Conferência de Saúde do Distrito Federal. Nesse evento, foram apontadas as deficiências na
Organização dos serviços de média e alta complexidades

Organização da Atenção Primária à Saúde

Fortalecimento de recursos humanos em saúde

Gestão do sistema de saúde
Construção e organização dos hospitais gerais em cinco cidades satélites; instalação da primeira unidade de terapia intensiva (UTI) no Distrito Federal Construção da farmácia e almoxarifado centrais; criação do Centro de Orientação Psicopedagógica para atendimento a portadores de necessidades especiais Reforma e construção de unidade básica de saúde no Plano Piloto, nas cidades satélites e em núcleos rurais

Criação da residência e internato médico; fundação de escola para a formação de auxiliares de enfermagem

Criação da Fundação Hospitalar do Distrito Federal e elaboração do Plano de Saúde de Brasília (Bandeira de Mello)

Fonte: Governo do Distrito Federal 22; Kaniak 23; Frejat 24; Silva 26; Carvalho 27; Scartezini 28; Melo 29. 


\begin{tabular}{|c|c|}
\hline & Ações \\
\hline Organização dos serviços de média e alta complexidades & $\begin{array}{l}\text { Ampliação e construção de três hospitais no Plano Piloto e cinco em cidades } \\
\text { satélites. Implantação da primeira unidade especializada no atendimento à saúde } \\
\text { mental no Distrito Federal. Criação de serviços voltados para a vigilância } \\
\text { epidemiológica e sanitária, além de laboratórios regionais para apoio diagnóstico }\end{array}$ \\
\hline Organização da Atenção Primária à Saúde & $\begin{array}{l}\text { Realização de campanhas para desratização, erradicação da varíola e da } \\
\text { poliomielite; controle da raiva, da hanseníase, da tuberculose, da doença de } \\
\text { Chagas, da esquistossomose e de doenças transmissíveis agudas; construção } \\
\text { de mais de quarenta unidades básicas de saúde em áreas urbanas e rurais de } \\
\text { cidades satélites e Plano Piloto. Realização da primeira experiência considerada } \\
\text { a semente do PSF pela Unidade de Saúde de Planaltina e pelo Grupo de } \\
\text { Integração Comunitária (prestação de "assistência médica, social e de } \\
\text { enfermagem" às famílias rurais por equipes multiprofissionais) implementada no } \\
\text { período de } 1974 \text { a } 1978\end{array}$ \\
\hline Fortalecimento de recursos humanos em saúde & $\begin{array}{l}\text { Ações voltadas para a formação, seleção e treinamento de pessoal para atuar em } \\
\text { cozinha, lavanderia e limpeza das unidades de saúde realizadas por quatro anos } \\
\text { consecutivos. Regulamentação do Programa de Residência Médica pela } \\
\text { Lei no. } 6.932 / 81 \text { e pela Comissão Nacional de Residência Médica criada } \\
\text { pelo MEC em } 1977\end{array}$ \\
\hline Gestão do sistema de saúde & $\begin{array}{l}\text { Elaboração e implantação de novo plano que previa a construção de unidades } \\
\text { básicas de saúde, com vistas à descentralização do atendimento, inspirado no } \\
\text { modelo de saúde adotado na Inglaterra e Alma Ata }\end{array}$ \\
\hline
\end{tabular}

PSF: Programa Saúde da Família; MEC: Ministério da Educação.

Fonte: Governo do Distrito Federal 22; Kaniak 23; Frejat 24; Silva 26; Carvalho 27; Scartezini 28; Melo 29; Lei nº $6.932 / 8130$.

gestão da SES-DF, a saber: (a) distorções na utilização das unidades básicas de saúde decorrentes da padronização do atendimento, contrapondose às diferenças na composição demográfica e epidemiológica da população; (b) autoritarismo na implantação do modelo de atenção, sem a participação da sociedade e de profissionais de saúde; (c) abandono dos princípios originais do plano de saúde; (d) predomínio da assistência hospitalar.

Com a implantação do Sistema Unificado Descentralizado de Saúde (SUDS), ocorreu a sobreposição das políticas nacional e local a qual possibilitará, nos anos seguintes, a criação das condições políticas para a proposição de mudanças na organização da atenção primária por meio do Programa Saúde em Casa (PSC). Data do mesmo período a criação de uma escola para a formação e a capacitação dos servidores da SES-DF, gestada no contexto de mudança do modelo de atenção. As dispersas instituições públicas de saúde são integradas à SES-DF (unidades do Instituto Nacional de Assistência Médica da Previdência Social, Hospital do Servidor da União e Centro de Reabilitação Sarah Kubitschek) no âmbito hospitalar, criando as condições para a construção futura do SUS-DF. Reordenam-se os recursos humanos oriundos das diferentes instituições de saúde, agregando-se os novos contratados em decorrência de concursos públicos. Contudo, a atenção primária não é priorizada (Tabela 3).

\section{- Quarto período: 1990 a 1998}

No período de 1990 a 1998, foram quatro os Secretários responsáveis pela política de saúde no Distrito Federal, com uma média de dois anos de permanência no poder. No plano nacional, a Constituição Federal de 1988 propunha a "saúde como direito de todos e dever do Estado", princípio regulamentado pelas Leis $n^{o} .8 .080^{1}$ e $n^{o} .8 .142^{25}$, publicadas em 1990. A repercussão desses instrumentos legais se faz sentir nas políticas públicas de saúde do Distrito Federal.

Esse foi um momento marcante para a implantação da Atenção Primária à Saúde no Distrito Federal. O relatório da II Conferência de Saúde do Distrito Federal enfatizou o distanciamento entre a rede básica e as ações relativas ao perfil epidemiológico da população adscrita, a centralização da gestão da política de saúde, o cresci- 
Síntese das principais ações de organização do sistema de saúde do Distrito Federal, Brasil, no período de 1983 a 1990.

\begin{tabular}{|c|c|}
\hline & Ações \\
\hline Organização dos serviços de média e alta complexidades & $\begin{array}{l}\text { Reformas da rede hospitalar atingindo praticamente todas as unidades de saúde } \\
\text { do Distrito Federal e reforço da política de saúde mental. Construção de um } \\
\text { centro de atenção psicossocial, em regime de hospital-dia, considerado o marco } \\
\text { na deshospitalização e na renovação da assistência psiquiátrica no Brasil } \\
\text { (junho/1987). Inauguração da Central de Radiologia e Hemocentro }\end{array}$ \\
\hline Organização da Atenção Primária à Saúde & $\begin{array}{l}\text { Reformas de unidades básicas de saúde e ênfase nos programas de vigilância } \\
\text { epidemiológica, imunização, assistência integral à saúde da mulher e da criança, } \\
\text { controle de doenças sexualmente transmissíveis e da síndrome de } \\
\text { imunodeficiência humana adquirida (AIDS) }\end{array}$ \\
\hline Fortalecimento de recursos humanos em saúde & $\begin{array}{l}\text { Revisão da política de recursos humanos objetivando a elevação da qualidade da } \\
\text { assistência, ensino e competência profissional, em todos os níveis. Realização de } \\
19 \text { concursos públicos em um ano, contemplando a quase totalidade das áreas do } \\
\text { setor. Criação da Revista de Saúde do Distrito Federal }\end{array}$ \\
\hline Gestão do sistema de saúde & $\begin{array}{l}\text { Instituição do SUDS no Distrito Federal, por meio do Convênio nº } 090 \text { de 30/ } \\
\text { set/1987. Elaboração de plano para o resgate da Atenção Primária à Saúde, } \\
\text { implantando a Gratificação de Atenção Básica, com suplementação de 30\% sobre } \\
\text { o salário-base do profissional de saúde lotado em Unidades Básicas de Saúde. } \\
\text { Realização da I Conferência de Saúde do Distrito Federal }\end{array}$ \\
\hline
\end{tabular}

SUDS: Sistema Unificado Descentralizado de Saúde.

Fonte: Governo do Distrito Federal 22; Kaniak 23; Frejat 24; Silva 26; Carvalho 27; Scartezini 28; Melo 29.

mento demográfico desordenado, a deterioração do padrão de vida da população, a redução dos recursos do setor saúde, a ausência de política de desenvolvimento de recursos humanos e a baixa resolubilidade da rede física de serviços de Atenção Primária à Saúde. Essa realidade reforçou a decisão governamental de instituir o PSC como estratégia de organização da Atenção Primária à Saúde. A proposta é implantada em parcerias com o Ministério da Saúde, recebendo investimentos na formação e na qualificação dos trabalhadores da saúde.

Embora existam evidências de avanços em termos de cobertura populacional pelo PSC, algumas limitações da proposta foram marcantes e podem explicar seus desdobramentos posteriores. Dentre essas, destacam-se: a duplicação de estruturas de atendimento, com funcionamento paralelo à rede de saúde tradicional, em residências alugadas, desarticuladas das atividades dos Centros e Postos de Saúde existentes; a contratação de trabalhadores da Estratégia Saúde da Família por meio de organização não-governamental, fora, portanto, dos quadros do serviço público; e a retribuição salarial muito acima dos valores pagos ao funcionalismo público, argumento recorrente para explicar a resistência dos servidores da SES-DF ao trabalho das equipes do PSC.
Esse modelo de organização da rede básica pautou-se pela correlação de forças entre os profissionais de saúde e a gestão da SES-DF no cotidiano do processo de trabalho, com fortes resistências dos servidores às mudanças, culminando com a não adesão/rejeição à proposta do PSC. Além disso, a mudança radical do governo no segundo ano de implantação do PSC provocou a brusca interrupção da iniciativa, com retorno ao modelo tradicional de atenção à saúde, registrando-se, no entanto, sucessivas tentativas de retomada nos anos seguintes (Tabela 4).

\section{- Quinto período: 1999 a 2007}

De 1999 a 2007, a SES-DF esteve liderada por três Secretários de Saúde, com uma média de permanência de 2,6 anos. A partir de 2005, assume a SES-DF o primeiro gestor não-médico.

Nos dois últimos períodos, especialmente a partir de 1999, a implantação da Estratégia Saúde da Família passou por mudanças na sua operacionalização, a saber: gratificação de $20 \%$ sobre o vencimento para servidores da SES-DF das equipes de Estratégia Saúde da Família; construção de trinta postos na área urbana para atuação dessas equipes; contratação dos demais profissionais para a completa composição do Programa Saúde da Família (PSF) pela mesma organização 


\begin{tabular}{|c|c|}
\hline & Ações \\
\hline \multirow[t]{5}{*}{ Organização dos serviços de média e alta complexidades } & Premiação para o primeiro Hospital Amigo da Criança de Taguatinga, pelo \\
\hline & UNICEF. Implantação de hospital especializado em pacientes crônicos. \\
\hline & Reforma e ampliação de outros oito hospitais. Instituição de programas de \\
\hline & assistência à saúde do idoso, do adolescente, de portadores de diabetes e à \\
\hline & saúde mental \\
\hline \multirow[t]{3}{*}{ Organização da Atenção Primária à Saúde } & Implantação do PSC como estratégia de reformulação do modelo de atenção à \\
\hline & saúde, atingindo 14 das 19 cidades do Distrito Federal, nas quais atuavam 278 \\
\hline & equipes de PSF em áreas urbana e rural \\
\hline \multirow[t]{4}{*}{ Fortalecimento de recursos humanos em saúde } & Realização de concurso para várias especialidades médicas; investimento em \\
\hline & treinamento de pessoal administrativo diretamente envolvido com o atendimento \\
\hline & ao público (recepcionistas, agentes de portaria, entre outros). Criação do pólo de \\
\hline & capacitação, formação e educação permanente para os profissionais de PSF \\
\hline \multirow[t]{3}{*}{ Gestão do sistema de saúde } & Elaboração do plano de Reformulação do Modelo de Atenção à Saúde no Distrito \\
\hline & Federal; implantação de uma política de melhoria da gestão financeira para o \\
\hline & SUS/DF; realização da II Conferência de Saúde do Distrito Federal \\
\hline
\end{tabular}

UNICEF: Fundo das Nações Unidas para a Infância; PSC: Programa Saúde em Casa; PSF: Programa Saúde da Família; SUS: Sistema Único de Saúde. Fonte: Governo do Distrito Federal 22; Kaniak 23; Frejat 24; Silva 26; Carvalho 27; Scartezini 28; Melo 29.

não-governamental do PSC. A Atenção Primária à Saúde passa a ser o marco das mudanças na política de saúde, a integrar a pauta de discussões entre profissionais de saúde, a ser objeto de disputas político-partidárias e a justificar a realização de parcerias público-privadas para a gestão do setor. No entanto, não foi capaz de alterar o funcionamento das unidades tradicionais de Atenção Primária à Saúde. Deve-se citar, ainda, o Plano de Conversão da Atenção Primária à Saúde no Distrito Federal para o período 2005-2008, que propõe a adoção dos princípios da Estratégia Saúde da Família para os centros de saúde tradicionais, prevendo-se a implementação paralela de equipes específicas para este fim, sem, todavia, mudanças nos demais níveis de atenção para a organização de uma rede de serviços condizente com a proposta (Tabela 5).

\section{Discutindo a trajetória da política de atenção básica do Distrito Federal na perspectiva neo-institucional}

A retrospectiva dos 47 anos de gestão da saúde no Distrito Federal mostra investimentos maciços de recursos financeiros e políticos na construção de hospitais. Evidencia, ainda, que as iniciativas para fortalecer a Atenção Primária à Saúde foram descontínuas e desarticuladas da rede de serviços de média e alta complexidades, com reduzida oferta de capacitação profissional, justificada pe- la necessidade de garantir a retaguarda da atenção hospitalar. Para a formação de profissionais - principais atores sociais e técnicos que respondem pelo cotidiano das práticas de saúde - foram implantados programas de pós-graduação na modalidade de residência, visando à formação de médicos especialistas para atendimento hospitalar. Somente nos últimos quatro anos o programa de residência incorporou outras categorias profissionais, tais como enfermagem, nutrição, serviço social, odontologia e especialidades médicas referentes à atenção primária.

Os Planos Bandeira de Mello e Jofran Frejat responderam pelas principais diretrizes para organização de ações e serviços de saúde no Distrito Federal, destacando-se como marcos que ainda vêm norteando a política de saúde e percorrendo uma trajetória que afetou o conjunto de opções de políticas públicas posteriores. A contradição entre o discurso em defesa da Atenção Primária à Saúde e a manutenção das metas de construção de novos hospitais apresentada a cada nova gestão, testemunha a forte característica de dependência da trajetória.

No que se refere à organização da rede de serviços, a despeito das inovações, a gestão da SES-DF optou por caminhos tradicionais, tais como: investimento nos hospitais como locus central do sistema de saúde; centralização da gestão financeira e de recursos humanos; uso de tecnologia de ponta; reforço na residência médica centrada na especialidade em detrimento dos demais pro- 
Síntese das principais ações de organização do sistema de saúde do Distrito Federal, Brasil, no período de 1999 a 2007.

\begin{tabular}{|c|c|}
\hline & Ações \\
\hline \multirow[t]{3}{*}{ Organização dos serviços de média e alta complexidades } & Reforma de cinco hospitais em cidades do Distrito Federal e Plano Piloto. \\
\hline & Realização de transplantes renais e hepáticos. Aquisição de equipamentos de \\
\hline & apoio a diagnósticos: ressonância magnética; tomógrafo e gama câmara \\
\hline \multirow[t]{6}{*}{ Organização da Atenção Primária à Saúde } & Substituição do PSC pelo PSF com mudanças na operacionalização, em \\
\hline & decorrência da solicitação de cancelamento pelo Ministério Público do Distrito \\
\hline & Federal que questionou o programa devido à contratação de trabalhadores sem \\
\hline & concurso público. Elaboração de proposta de plano de conversão da rede \\
\hline & tradicional de Atenção Primária à Saúde para atuar nos moldes da Estratégia \\
\hline & Saúde da Família \\
\hline \multirow[t]{3}{*}{ Fortalecimento de recursos humanos em saúde } & Criação da Fundação de Ensino e Pesquisa e da Escola Superior de Ciências da \\
\hline & Saúde, vinculadas a SES-DF, e do curso de medicina. Implantação do Plano de \\
\hline & Cargos, Carreiras e Salários dos servidores da SES-DF \\
\hline \multirow[t]{4}{*}{ Gestão do sistema de saúde } & Implantação do processo de compra de serviços de saúde do setor privado (leitos \\
\hline & de UTI, de unidades coronarianas e de reabilitação do aparelho motor). \\
\hline & Elaboração do Plano Diretor de Regionalização em 2002, pré-requisito para a \\
\hline & qualificação da NOAS-SUS 01/02 \\
\hline
\end{tabular}

PSC: Programa Saúde em Casa; PSF: Programa Saúde da Família; SES-DF: Secretaria de Estado da Saúde do Distrito Federal; NOAS-SUS 01/02: Norma Operacional de Assistência à Saúde.

Fonte: Governo do Distrito Federal 22; Kaniak 23; Frejat 24; Silva 26; Carvalho 27; Scartezini 28; Melo 29.

fissionais. Mesmo quando integrou a agenda da política de saúde, a Atenção Primária à Saúde foi considerada acessória ao atendimento hospitalar, objetivando "desafogá-lo".

Em relação à gestão da política de saúde, há sobreposição dos níveis nacional e local na definição das diretrizes políticas. Isso traz dificuldades ao Distrito Federal para responder às demandas da população, para atender às exigências do Ministério da Saúde, bem como para a prestação de contas aos mecanismos de controle social e governamental. Essa dificuldade se manifesta nas freqüentes trocas de gestores e nas oscilações bruscas da política de saúde, que se exaure tentando responder às diretrizes nacionais, em detrimento de propostas mais condizentes com as demandas locais. Tal situação se explica também pela baixa participação política que caracteriza o sistema político do Governo do Distrito Federal, no qual prepondera a centralização no processo decisório. No caso da política de saúde, a inexistência de municípios torna a gestão da SES-DF pouco permeável ao debate político com os demais níveis de governo e com as estruturas instituídas de controle social, como os Conselhos e as Conferências de Saúde.

Talvez este seja um momento propício para a proposição de novos planos para a organização dos serviços de saúde no Distrito Federal, plane- jados e estruturados de forma participativa, coerente com os princípios do SUS e com as necessidades atuais da população, incluindo a Região do Entorno. Uma proposta que associe a organização de redes de cuidados e que contemple a Atenção Primária à Saúde com todo o seu potencial reordenador do modelo assistencial, devidamente articulado aos demais níveis de atenção. Enfim, um modelo que precisa ser forjado para ser resolutivo, sustentável e inclusivo, capaz de responder às conseqüências da explosão demográfica que o Distrito Federal vem enfrentando ao longo da sua trajetória de 48 anos. 


\section{Resumo}

Este artigo analisa a trajetória da política de atenção básica à saúde no Distrito Federal, Brasil, a partir do marco teórico do neo-institucionalismo histórico, identificando as conformações e as tendências predominantes nas gestões da Secretaria de Estado da Saúde (SES-DF) no período de 1960 a 2007. O estudo sinaliza que a política de saúde do Distrito Federal apresenta características de dependência da trajetória dos planos de saúde originais na definição de prioridades e metas, bem como na implementação do sistema de saúde. Essa influência, agregada à centralização dos processos decisórios e à limitada participação política, pode contribuir para situar a atenção básica como acessória ao atendimento hospitalar, destituindo-a do seu potencial de produzir mudança no modelo assistencial.

Política de Saúde; Atenção Primária à Saúde; Sistema Único de Saúde

\section{Colaboradores}

L. B. D. Göttems elaborou o artigo. M. S. N. Evangelista e M. R. G. M. Pires colaboraram na elaboração e na revisão. A. F. M. Silva e P. A. Silva colaboraram na concepção e na redação do artigo.

\section{Referências}

1. Brasil. Lei Federal no. 8.080/90. Dispõe sobre as condições para a promoção, proteção e recuperação da saúde, a organização e o funcionamento dos serviços correspondentes e dá outras providências. Diário Oficial da União 1990; 19 set.

2. Göttems LBD, Pires MRGM, Guilhem D, Alves ED, Martins CMF. Relatório final de pesquisa: análise da relação dos serviços de média complexidade com a rede básica de saúde do DF e entorno: buscando prioridades para ampliar acessos e resolubilidades. Brasília: Universidade de Brasília/Universidade Católica de Brasília/Universidade Federal de Minas Gerais; 2008. http://nesprom.universidadevirtual.br/ (acessado em 22/Jul/2008).

3. Ministério da Saúde. Portaria n ${ }^{\circ}$. 648/2006, que estabelece a Política Nacional de Atenção Básica. Brasília: Ministério da Saúde; 2006.

4. Conill EM. Políticas de atenção primária e reformas sanitárias: discutindo a avaliação a partir da análise do Programa Saúde da Família em Florianópolis, Santa Catarina, Brasil, 1994-2000. Cad Saúde Pública 2002; 18 Suppl:191-202.

5. Viana ALA, Rocha JSY, Elias PE, Ibañez N, Novaes MHD. Modelos de atenção básica nos grandes municípios paulistas: efetividade, eficácia, sustentabilidade e governabilidade. Ciênc Saúde Coletiva 2006; 11:577-606.
6. Departamento de Atenção Básica, Secretaria de Atenção à Saúde, Ministério da Saúde. Saúde da família no Brasil: uma análise de indicadores selecionados: 1998-2004. http://dtr2004.saude.gov. $\mathrm{br} / \mathrm{dab} / \mathrm{caa} /$ publicacoes.php (acessado em 22/ Jul/08).

7. Elias PE, Ferreira CW, Alves MCG, Cohn AKV, Escrivão JA, Gomes A, et al. Atenção Básica em Saúde: comparação entre PSF e UBS por estrato de exclusão social no Município de São Paulo. Ciênc Saúde Coletiva 2006; 11:633-41.

8. Governo do Distrito Federal. Plano Diretor de Regionalização. Brasília: Secretaria de Estado de Saúde do Distrito Federal; 2005.

9. Demo P. Metodologia do conhecimento científico. São Paulo: Editora Atlas; 2000.

10. LudkeM,AndréM.Métododecoletadedados:observação, entrevista e análise documental. In:Ludke M, André MEDA, organizadores. Pesquisa em educação: abordagens qualitativas. São Paulo: EPU; 1980. p. 25-44.

11. Franco MLPB. Análise de conteúdo. 2a Ed. Brasília: Líber Livro Editora; 2005.

12. Bonafont LC. Redes de políticas públicas. Madrid: Centro de Investigaciones Sociológicas; 2004.

13. Souza C. "Estado do campo" da pesquisa em políticas públicas no Brasil. Revista Brasileira de Ciências Sociais 2003; 18:15-20. 
14. Flexor G, Leite SP. Análise de políticas públicas: breves considerações teórico-metodológicas. http://www.sep.org.br/artigo (acessado em 01/ Nov/2007).

15. Hall P, Taylor R. As três versões do neo-institucionalismo. Lua Nova 2003; 58:193-223.

16. Theret B. As instituições entre as estruturas e as ações. Lua Nova 2003; 58:225-54.

17. Frey K. Políticas públicas: um debate conceitual e reflexões referentes à prática da análise de políticas públicas no Brasil. Planej Polít Públicas 2000; 21:211-59.

18. North D. Institutions, institutional change and economic performance. Princeton: Cambridge University Press; 1991

19. Pierson P. Politics in time: history institutions and social analysis. Princeton: Princeton University Press; 2005.

20. Heller C. Path-dependence, lock-in e inércia. In: Pelaez V, Szmrecsányi T, organizadores. Economia e inovação tecnológica. São Paulo: Editora Hucitec/Ordem dos Economistas do Brasil; 2006. p. 260-84.

21. Gerschman S, Santos MAB. O Sistema Único de Saúde como desdobramento das políticas de saúde do século XX. Revista Brasileira de Ciências Sociais 2006; 21:177-227.

22. Governo do Distrito Federal. Programa Família Saudável no Distrito Federal: plano de conversão da atenção primária à saúde no DF sob a estratégia de saúde da família - 2005 a 2008. Brasília: Secretaria de Estado da Saúde do Distrito Federal; 2005.
23. Kaniak CEA. Plano Geral da Rede Médico-Hospitalar para o Distrito Federal de Henrique Bandeira de Mello. Rev Saúde Dist Fed 1999; 10:52-3.

24. Frejat J. Sistema de saúde do DF: modelo para o país. Rev Saúde Dist Fed 1999; 10:53-9.

25. Brasil. Lei Federal no ${ }^{\circ}$. 8.142/90. Dispõe sobre a participação da comunidade na gestão do Sistema Único de Saúde (SUS) e sobre as transferências intergovernamentais de recursos financeiros na área da saúde e dá outras providências. Diário Oficial da União 1990; $28 \mathrm{dez}$.

26. Silva E. A saga da Fundação Hospitalar no Distrito Federal: e a medicina da poeira e da lama. Brasília: Gráfica e Editora Copacabana; 2000.

27. Carvalho AG. Programa Saúde da Família resgata compromisso social do início de Brasília. Rev Saúde Dist Fed 1999; 10:60-5.

28. Scartezini M. Hospital Juscelino Kubitschek de Oliveira (Ex-hospital do IAPI). Rev Saúde Dist Fed 1999; 10:66-70.

29. Melo HB. Plano Geral da Rede Médico-Hospitalar de Brasília. Revista do Serviço de Saúde Pública 1959; XI(1).

30. Brasil. Lei Federal $n^{\circ}$. 6.932/81. Dispõe sobre as atividades do médico residente e dá outras providências. Diário Oficial da União 1981; 9 jul.

Recebido em 11/Set/2008

Versão final reapresentada em 10/Dez/2008

Aprovado em 26/Jan/2009 